\title{
SEMISIMPLE COMPLETELY DISTRIBUTIVE LATTICES ARE BOOLEAN ALGEBRAS
}

\author{
M. S. LAMBROU
}

ABSTRACT. Semisimple completely distributive lattices are Boolean algebras.

The purpose of this note is to prove the result of the title, where, throughout, the term lattice will be taken to mean complete lattice with 0 and 1. If $L$ is a lattice then a subset $M$ of $L$ is said to be a semi-ideal of $L$ if for all $a \in M$ and $b \in L$ such that $b \leqslant a$ then $b \in M$. An ideal $M$ of $L$ is a semi-ideal with the property that if $a, b \in M$ then so does their span $a \bigvee b$. If the intersection of all maximal (proper) ideals of $L$ is $0, L$ is said to be semisimple, and $L$ is called completely distributive if for all $a$ in $L$,

$$
a=\bigvee \cap\{M: M \text { is a semi-ideal and } a \leqslant \bigvee M\} \text {, }
$$

where $\cap$ denotes set theoretic intersection. This definition of completely distributive lattice is not the original one [1], but was proved to be equivalent to it by Raney [5]. For each element $a$ of the complete lattice $L$ we write

$$
a_{-}=\bigvee\{b \in L: a \leqslant b\} \text {. }
$$

It is shown in Longstaff [4] using Raney's characterisation that a necessary and sufficient condition for a complete lattice to be completely distributive is that for all $a$ in $L$,

$$
a=\bigvee\left\{b \in L: a \leqslant b_{-}\right\}
$$

Examples of semisimple lattices are Boolean algebras. This follows from the fact that an ideal $M$ of $L$ is maximal if and only if for each $a$ in $L$, either $a$ or its Boolean algebra complement $a^{\prime}$, but not both, are in $M$ (see [2, p. 71]). An example of a completely distributive lattice is any complete atomic Boolean algebra [1, p. 95]. It is a result of Tarski that a Boolean algebra is completely distributive if and only if it is atomic [1, p. 95]. The theorem below implies the following strengthening of Tarski's result: A complete lattice with 0 and 1 is an atomic Boolean algebra if and only if it is semisimple and completely distributive.

LEMmA. If $L$ is a completely distributive lattice such that $a \wedge a_{-}=0$ for all

Received by the editors April 1, 1977.

AMS (MOS) subject classifications (1970). Primary 02J05, 06A23, 06A35, 06A40; Secondary 47A15.

Key words and phrases. Maximal ideals, semisimple, completely distributive, Boolean algebras, atomic.

() American Mathematical Society 1978 
$a$ in $L$ with $a_{-} \neq 1$, then $L$ is a complete atomic Boolean algebra.

Proof. We first prove that a nonzero element $a$ of $L$ is an atom if and only if $a_{-} \neq 1$. Indeed if $a_{-} \neq 1$ then for any $b<a$ (where $<$ is strict) we have $a \nless b$. Hence by the definition of $a_{-}, b \leqslant a_{-}$, therefore $b \leqslant a \wedge a_{-}=0$ and so $b=0$. Conversely,

$$
a \nless b \Rightarrow a \wedge b<a
$$

and, hence, if $a$ is an atom, $a \wedge b=0$. By complete distributivity

$$
\begin{aligned}
a \wedge a_{-} & =a \wedge(\bigvee\{b \in L \mid a \leqslant b\}) \\
& =\bigvee\{a \wedge b \mid b \in L, a \leqslant b\}=\bigvee\{0\}=0
\end{aligned}
$$

and so $a_{-} \neq 1$.

From the above discussion we have, for all $a$ in $L$,

$$
a=\bigvee\left\{b \in L \mid a \leqslant b_{-}\right\}
$$

Notice that $a k b_{-}$implies that, in particular, $b_{-} \neq 1$, that is, $b$ is an atom and so this equality modifies to

$$
a^{\prime}=\bigvee\{b \in L \mid b \text { is an atom contained in } a\},
$$

which in turn implies the lattice is atomic.

For each $c$ in $L$ define

$$
c^{\prime}=\bigvee\{b \in L \mid b \text { is an atom not contained in } c\} .
$$

It is easy to check, using the complete distributivity assumption, that $c \wedge c^{\prime}=0$ and $c \vee c^{\prime}=1$, and so $c^{\prime}$ is a complement of $c$. By distributivity of $L c^{\prime}$ is unique and so it is the Boolean algebra complement of $c$. This completes the proof of the Lemma.

TheOREM. A complete lattice with 0 and 1 is an atomic Boolean algebra if and only if it is semisimple and completely distributive.

Proof. Let a complete lattice $L$ be semisimple and completely distributive. We first prove that for any maximal ideal $M$ of $L$ and any $a \in L$ such that $a_{-} \neq 1$, we have $a \wedge a_{-} \in M$. Indeed, if $a \in M$ then by definition of an ideal, so is $a \wedge a_{-}$, so we can assume $a \notin M$. If $m$ is an arbitrary element of $M$ we cannot have $a \leqslant m$ because then $a$ would belong to $M$, hence $a \nless m$, which implies $m \leqslant a_{-}$. This last inequality is true for all $m \in M$ and, hence,

$$
M \subseteq\left\{c \in L \mid c \leqslant a_{-}\right\} .
$$

The set on the right is trivially an ideal of $L$ and as $a_{-} \neq 1$, it is proper. By maximality of $M$ we have

$$
M=\left\{c \in L \mid c \leqslant a_{-}\right\} .
$$

As $a_{-} \leqslant a_{-}$we get that $a_{-} \in M$, which implies $a \wedge a_{-} \in M$ (an ideal) as claimed.

The maximal ideal $M$ was arbitrary, hence we conclude that if $a_{-} \neq 1$, $a \wedge a_{-}$is in the intersection of all maximal ideals of $L$. The semisimplicity of 
$L$ now shows that $a \wedge a_{-}=0$, and so from the Lemma, $L$ is a complete atomic Boolean algebra.

Conversely, if $L$ is a complete atomic Boolean algebra then by Tarski's theorem mentioned above it is completely distributive, and it is certainly semisimple because all Boolean algebras are, completing the proof of the theorem.

REMARK. In the special case of lattices of subspaces of a Hilbert space the above theorem is a corollary of a characterization of complete atomic Boolean algebras in terms of the algebra of all operators that leave the lattice invariant (see [3]). The motivation for the present work was to establish the same result in the context of general lattice theory and so independent of the spatial properties of Hilbert space.

\section{REFERENCES}

1. G. Birkhoff, Lattice theory, Amer. Math. Soc. Colloq. Publ., vol. 25, Amer. Math. Soc., Providence, R. I., 1940.

2. P. R. Halmos, Lectures on Boolean algebras, Van Nostrand Math. Studies, 1963.

3. M. S. Lambrou, Complete atomic Boolean lattices, J. London Math. Soc. (2) 15 (1977), 387-390.

4. W. E. Longstaff, Strongly reflexive lattices, J. London Math. Soc. (2) 11 (1975), 491-498.

5. G. N. Raney, A sub-direct union representation for completely distributive lattices, Proc. Amer. Math. Soc. 4 (1953), 518-522.

Department of Mathematics, King's College, London WC2, England

Current address: Moshonision 42, Athens (823), Greece 